Kontraktionsstärke des Herzens bedeutend herabgesetzt wird. Die Wirkung tritt auch schon auf kleine Dosen ein $(1,0)$. Die Geschwindigkeit, mit der die Wirkung eintritt, ist groB, da schon nach 3 Stunden eine fast maximale Abnahme der Hubhöhe festzustellen ist. Ein Aussetzen der Kalktherapie läßt schon nach 24 Stunden ein fast vollkommenes Abklingen der Kalkwirkung erkennen. Ob die abnehmende Kalkwirkung schon viel früher bemerkbar ist, läßt sich mit Sicherheit nicht aus den Resultaten schließen. - Infolge des stets gleichgerichtet auftretenden Wirkungsmodus des Serums bei Kalkbehandlung bzw. Absetzen derselben kann man den Schluß ziehen, da $B$ als Folge der Kalktherapie in der Zusammensetzung des Blutes eine Veränderung vor sich geht, von deren Existenz man bisher nichts wuBte. Welcher Art diese Veränderung ist, läßt sich nicht ohne nähere Blutanalyse entscheiden; es könnte sich um eine direkte Abnahme des Blutkalkgehaltes handeln und fände dann eine Stütze in den Befunden von Neurath und Katzenellenbogen, die ,in einigen Fällen eine Verminderung des Blutkalkgehaltes" fanden. Andererseits wäre an eine vermehrte Aktivierung von Kalium, das eine herabsetzende inotrope Wirkung besitzt, oder gewisser organischer Substanzen von ähnlicher biologischer Wirkung zu denken 1 ). Wenn ich auf dieWirkung derKalktherapie schon einen Schluß ziehen kann, so ist es wohl vor allem der, daß wir mit Kalk nicht die Kalkarmut des Blutes kausal behandeln und daß die Wirkung eine sehr flüchtige ist, so $d a B$ nach 24. Stunden fast keine Spuren mehr nachweisbar sind. Daraus ergibt sich die Notwendigkeit, den Körper mit kurz nacheinander verabfolgten Kalkgaben zu überschwemmen (BLÜHDORN).

Wenn man nicht annimmt, daß eine starke Calciumchloridfütterung trotz nachweisbarer Wirkung auf das Nervensystem den Kalkgehalt des Serums herabsetzt, so muB der Kalk in diesem Serum in anders wirksamer Form enthalten sein, als wenn ich ihn in vitro in Form von Calciumchlorid oder Calcium lacticum zusetze. Damit ist es aber durchaus diskutabel geworden, ob die Kalkarmut des Blutes allein oder zum mindesten als quantitative Erscheinung schuld an der Tetanie ist. Die inzwischen erschienenen Erwägungen von FreUdenberg und György bewegen sich in gleichem Sinne.

\section{UNTERSUCHUNGEN ÜBER DIE FERMENTE DES PANKREAS VOR UND NACH INJEKTION VON ÄTHER INS DUODENUM.}

\section{Von}

Dr. Eva Langanke.

Aus der Medizinischen Universitätsklin k zu Königsberg i. Pr. (Direktor: Geheimrat Prof. Dr. MATTHES.)

KATSCH und FRIEDRICH ${ }^{2}$ ) haben kürzlich mitgeteilt, daß man durch Injektion von Ather ins Duodenum mittels der Duodenalsonde einen an Pankreasfermenten reichen Saft mit relativ wenig "Lebergalle" erhalten kann. An 20 Kranken

1) Diese theoretischen Ergebnisse der biologischen Untersuchung werden alle widerlegt durch den klinischen Befund, der ein sofortiges Zurückgehen der elektrischen Ubererregbarkeit ergibt. Es bliebe demnach nur übrig anzunehmen, daB das Calcium, weiches bei direktem Zusatz eine erhöhte inotrope Wirkung am Froschberzen besitzt, ein anderes ist, als das $\mathrm{Ca}$ nach enteraler Einverlejbung von Calciumchlurid.

3) KATSCH and FRIEDRICH. Klin. Wochenschr. Nr. 3. Igz2.

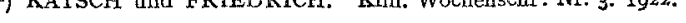

der medizinischen Klinik habe ich diese Angabe nachuntersucht.

Die Einhornsche Duodenalsonde wurde den Patienten nüchtern in der üblichen Weise eingeführt. Nachdem alkalisch reagierende ,Lebergalle“ geflossen war, wurden Erwachsenen $4-5 \mathrm{ccm}$, Kindern je nach dem Alter $1-3 \mathrm{ccm}$ ScherINGs Narkosenäther durch die Sonde hindurch ins Duodenum injiziert. Unangenehme Zwischenfälle wurden dabei nie beobachtet. Nur bei Patienten mit besonderer Labilität der Vasomotoren traten öfter kurz anhaltende rauschähnliche Zustände auf. Nach der Injektion dauerte es ungefähr 5-1o Minuten bis sich ein dünnflüssiger, blaßgelber, fast farbloser Saft entleerte. In den meisten. Fällen brauchte nicht aspiriert zu werden, es genügte schon die Heberwirkung. In 5 Fällen war vor und nach der Ätherinjektion keine Differenz in bezug auf die Farbe vorhanden. In 8 Fällen floß ungefähr I5-20 Minuten, nachdem sich der dünnflüssige, schwach gelb gefärbte Inhalt entleert hatte, eine tief dunkelbraune als ,Gallenblasengalle" anzusprechende Flüssigkeit.

Die vor und nach der Ätherinjektion gewonnenen Portionen wurden unverdünnt in Reihenversuchen gesondert auf die 3 wichtigsten Pankreasfermente untersucht. Das proteolytische Ferment, das Trypsin, wurde nach der FuLD-GrossGEMUTH $^{1}$ ) und das lipolytische Ferment, das Steapsin, mit der Kanitzschen Olemulsion angesetzt. Der Trypsinversuch wurde einstündig, der Diastase- und der Lipaseversuch wurden je 24stündig alle $3 \mathrm{im}$ Thermostaten ausgeführt.

Die vorstehende Ubersichtstabeile zeigt die Resultate der von uns untersuchten 20 Patienten.

Die Einheiten des Trypsins ergaben in 13 Fällen, die der Diastase in 16 Fällen und die der Lipase in 17 Fällen nach der Atherinjektion höhere Werte. Der Gehalt der Duodenalflüssigkeit vor und nach der Atherinjektion war an Trypsin in 6 Fällen, an Diastase in 4 Fällen und an Steapsin in nur I Fall der gleiche geblieben. Ein niedrigerer Wert nach der Injektion fand sich bei dem proteolytischen Ferment in I Fall, bei dem lipolytischen Ferment in 2 Fällen, bei dem diastatischen Ferment in keinem Fall. Die Ansicht von Bond und Salomon ${ }^{2}$ ), daß Anderungen des Fermentgehaltes alle Pankreasfermente gleichmäßig betrifft, läßt sich also nach unseren Resultaten nicht bestätigen. Darauf, daß die Erhöhung oder Erniedrigung der 3 Fermente keine RegelmäBigkeiten erkennen läßt, haben schon IsAAC-KRIEGER ${ }^{3}$ ) wie auch KATSCH und FRIEDRICH (1. c.) hingewiesen. Die 4 Fälle von schwerem Diabetes mellitus (s. Tabelle) zeigen auffallend niedrige Werte

1) WOHLGEMUTH, Grundriß der Fermentmethoden. Berlin rgr3.

8) BONDI und SALOMON, Beibl. z. d. Mitt. d. Ges. f. inn. Med. u. Kinderheilk., Wien. I2, I913. S. 156 .

") ISAAC-KR IEGER, Zeitschr. f. klin. Med. 92. I9zx. S. 259. 
für Trypsin und Steapsin. Auch bei einem Fall von Morbus Basedowi ist der Wert für das lipolytische Ferment stark herabgesetzt. In unseren Fällen von Subacidität und Achylia gastrica war keine abnorme Erniedrigung der Fermentwerte zu registrieren.

v. FALKENHAUSEN ${ }^{1}$ ) sieht allenfalls als noch unterste physiologische Grenze für Trypsin 200 Einheiten an und bezeichnet 300-400 Einheiten als Durchschnittswerte. Nach unseren Untersuchungen möchten wir uns der Ansicht IsAAC-KRIEGERS (l. c.) zuneigen, der als untersten Grenzwert für Trypsin I25 Einheiten, für Diastase I6I Einheiten und für Lipase $i, 0$ bis höchstens $2,4 \mathrm{ccm}$ (in $\mathrm{n} / 10 \mathrm{NaOH}$ titriert) ansieht.

Jedenfalls schwanken die Angaben über die Normalwerte der Fermenteinheiten noch zu sehr, als daß man aus den Resultaten der Fermentreaktionen klinisch sichere diagnostische Schlüsse ziehen könnte. Nur starker Herabsetzung der Fermenteinheiten im Duodenalsaft darf man, wie schon KATSCH und Friedrich (1. c.) und GANG und KLEIN ${ }^{2}$ ) betont haben, einige diagnostische Bedeutung zusprechen. Fehlen alle 3 Fermente im Duodenalsaft, auch nach Äthereinspritzung, so deutet das auf eine Insuffizienz der äußeren Pankreassekretion; einen Anhalt, welcher Art diese Störung sei, kann man jedoch daraus gicht gewinnen.

Zusammenfassung: Die von KATSCH und FrIEDRICH (1. c.) angegebene Methode ist eine bequeme Handhabe zur Erlangung von pankreasfermentreichem Duodenalsaft. Die Injektion der wenigen Kubikzentimeter Äther ist ohne Schaden für den Patienten. Fehlen auch nach der Injektion von Ather die Fermente in der Duodenalflüssigkeit, so spricht das für eine Funktionsuntüchtigkeit der äußeren Sekretion des Pankreas.

\section{ÜBER NIERENSCHUSSVERLETZUNGEN³).}

$$
\text { Von }
$$

\section{Dr. KuRT SCheyer, Assistenzarzt.}

Aus der chirurgischen Abteilung des Allerbeiligen-Hospitals Bresian. (Primärarzt: Prof. Dr. A. Tietze.)

Trotz der ungeheuren Fülle aller Arten von Schußverletzungen im Kriege ist die Zahl der berichteten Nierenschüsse eine auffallend kleine. Wenn unter Io-20 ooo Verwundungen manche Beobachter in den Feld- und Kriegslazaretten nur einige wenige Nierenschüsse sahen, so geben die an diesem Material angestellten Beobachtungen ebensowenig wie bei den Bauchschüssen ein wahrheitsgetreues Bild über die Schwere und die Prognose dieser Verletzungen. Denn, wie man als Truppenarzt sehen konnte, bleibt die große Zahl der Schwerstverletzten auf dem Schlachtfeld selbst oder stirbt auf dem Transport nach hinten. Und zu ihnen gehören sehr viele Nierenschüsse, sei es, daß die großen Gefäße am Hilus zerrissen sind oder schwere Verletzungen der Nachbarorgane, besonders der Bauchhöhle, stattgefunden haben. Oft auch mag die Verletzung letzterer so im Vordergrunde des klinischen Bildes gestanden haben, daß die Mitverletzung der Niere gar nicht genügend bewertet wurde. Diese relative Seltenheit der NierenschuBverletzungen, erst recht in der Friedenschirurgie, berechtigt wohl zur kurzen Mitteilung einiger in letzter Zeit im Allerheiligen-Hospital beobachteter Fälle.

Reine, isolierte Nierenschüsse sind bei đer versteckten Lage dieser Organe eine ganz große Seltenheit. Meist handelt es sich dabei um matte Granat- oder Minensplittersteckschüsse, die von hinten kommen, während die mit größerer Durchschlagskraft begabten Infanteriegeschosse nur in Form von Streifschüssen isoliert die Niere - und zwar besonders die rechte, tieferstehende - treffen können. Solche Granatsplittersteckschüsse können, wenn es sich dabei nicht um schwerere Schädigungen der Niere handelt, in der Folge zu paranephritischen oder Nierenabscessen mit Eiterung in die ableitenden Harnwege bzw. Pyelitis Veranlassung geben. $\mathrm{DaB}$ sie sich in einzelnen Fällen auch inkrustieren und voll-

1) v. FALKenhaUSen, Arch. f. exper. Pathol. u. Pharm. 92 5. x73.

?) GANG und KLEIN Med Klin, IgIt $\mathrm{Nr}$. I8.

8) Nach einem in der Schles. Geselischaft für vaterländische Kultur in Breslau ge balteven Vortrage. ständig, auch auf dem Röntgenbilde, die Symptome eines Nierensteines-machen können, lehrt ein Fall, den ich vor kurzem aus der Abteilung von Herrn Prof. Gotrstein in Breslau veröffentlichen konnte, und der 3 Jahre nach der Verletzung zur Extraktion des inkrustierten Granatsplitters durch Pyelotomie Veranlassung gab $^{\mathbf{1}}$.

Häufiger werden bei Nierenschüssen andere Organe, am häufigsten die der Bauchhöhle, mitverletzt. Hier handelt es sich meist um Durchschüsse der Niere, die je nach der Größe und Durchschlagskraft der Geschosse und der Entfernung,, aus der sie kommen, die Gestalt der Nierenwunde bedingen. Bei den modernen rasanten, aus großer Entfernung kommenden Gewehrgeschossen kommen daher auch glatte, einfache, kalibergroße Wunden vor, die wohl die GroBzahl der ohne Operation geheilten Nierenschüsse ausmachen, deren Vorkommen aber von manchen Autoren lange Zeit bezweifelt wurde. Um einen solchen einfachen Lochschuß der Niere muß es sich bei folgender Beobachtung aus der Privatpraxis von Herrn Prof. TiETze gehandelt haben.

Ein Mann wird während der oberschlesischen Kämpfe durch ein Gewehrgeschoß verletzt, das in der Mamillarlinie im linken Oberbauch dicht unter dem Rande des dort befindlichen Rippenknorpels eindringt und den Körper, etwa drei querfingerbreit von der Mitte der Wirbelsäule entfernt, dicht unter der I2. Rippe, verläBt. Pat. kollabiert nicht und wird $40 \mathrm{~km}$ weit im Auto nach Oppeln transportiert. Hier zeigt er 8-Io Stunden nach der Verletzung einen ruhigen Puls, keinerlei Peritonealerscheinungen, keine Zeichen einer Thoraxverletzung und als einziges Symptom tiefblutigen Urin, der zusammen mit der Richtung des Schußkanals die Diagnose auf Nierenschuß stellen ließ. Bei dem Fehlen jeglicher peritonealer oder sonstiger bedrohlicher Erscheinungen konnte von einer Operation Abstand genommen werden, und Patient ist ohne diese vollständig geheilt worden.

Der Fall ist in mancher Hinsicht bemerkenswert. Zunächst muß es als eine große Seltenheit bezeichnet werden, daß es bei einem intraperitonealen NierenschuB zu keiner Verletzung eines Bauchorganes gekommen ist. Immerhin sind solche Verletzungen, die KữTNER als ,Glücksschüsse". bezeichnet hat, und bei denen es sich offenbar tatsächlich um eine Verdrängung des Magendarms durch ein aus weiter Ferne kommendes Geschoß handelt, gerade im Oberbauch mehrfach beobachtet worden. In unserem Falle muB das Geschoß dicht unterhalb der Leber eingedrungen sein und oberhalb der kleinen Kurvatur des Magens vorbei den $1 \mathrm{k}$. oberen Nierenpol durchbohrt haben. Aber auch bei dieser Annahme ist das Fehlen aller peritonealen Reizerscheinungen bei einem intraperitonealen Nierenschu $\beta$ sehr auffallend. Fehlen diese doch sonst auch bei extraperitonealen Nierenverletzungen, offenbar durch das retroperitoneale Hämatom ausgelöst, fast nie. Hier stützte sich also die Diagnose des Nierenschusses - außer auf die Schußrichtung - nur auf die Hämaturie. Und doch kann auch dieses wichtige Symptom in einzelnen Fällen zeitweise fehlen, sei es, daB der Ureter abgerissen ist, der Nierenriß nicht bis ins Nierenbecken reicht oder der Ureter bereits durch ein Blutgerinnsel verstopft ist. Mikroskopisch freilich werden auch dann Blutbestandteile aachweisbar sein.

Im Gegensatz zu diesen recht seltenen Lochschüssen ist der Typus des Nierendurchschusses charakterisiert durch eine ausgedehnte Zertrümmerung des Nierengewebes, das gewöhnlich zahllreiche vom Schußkanal ausgehende Risse und Sprünge aufweist. Denn in dem weichen, von einer derben Kapsel eingeschlossenen Parenchym kommt es beim Auftreffen eines Geschosses aus geringerer oder mittlerer Entfernung zu einer Sprengwirkung, die vielfach, wie schon KüsTER gezeigt hat, durch die hydraulische Wirkung der in ihr enthaltenen Flüssigkeit bis zur völligen Zertrümmerung und zum Abriß des Or. ganes führen kann. Zu einer solchen schweren Zertrümmerung kam es in einem anderen kürzlich im Allerheiligen-Hospital beobachteten, Fall von Leber-Nierenschuß.

Einschuß etwa in der vorderen Axillarlinie, 3-4 Querfinger oberhalb des Rippenbogens durch Revolverkugel aus Entfernung von wenigen Metern. In etwa gleicher Höhe am Rücken Haut suggilat als Zeichen des vermutlichen Sitzes des Geschosses. Wegen der bedrohlichen Erscheinungen einer intraperitonealen Blutung

1) Deutsche Zeitschr. f. Chirurg. Bd. 166, S. 345 . 